\title{
Modelling Binary, Knudsen and Transition Regime Diffusion Inside Complex Porous Media
}

\author{
G.L. Vignoles
}

Laboratoire des Composites Thermostructuraux, UMR 47 CNRS-SEP, Université Bordeaux 1, 3, allée La Boëtie, Domaine Universitaire, 33600 Pessac, France

\begin{abstract}
The problem of gaseous diffusion inside complex porous media arises in the modeling of many chemical processes (c.g. Chemical Vapor Infiltration (CVI), heterogeneous catalysis in porous catalysts, filtration, etc...). A program computing effective diffusivities in the bulk, Knudsen and transition regimes has been designed and tested, which uses a Monte-Carlo mean-square-displacement algorithm. The porous medium has been represented from a special interpretation of a computer 3D discretized image. Simulations were carried out in a typical case of complex structured porous medium : a stacking of tissues (e. g. 2D woven fiber preform for CVI-densified composite materials). The results are presented as tortuosity factors, i.e. deviations from an equivalent medium made of straight cylindrical pores. The evolution of the diffusivities with the geometrical parameters of the tissues, and with the stacking mode has also been studied. It appears that the perpendicular diffusivity is closely rejated to the proportion of matching holes between different layers of tissue. The intermediate regime appears for Knudsen numbers lying between 100 and $10^{-1}$. In this domain, the Bosanquet formula only gives a good description if the Knudsen number is multiplied by a factor $\gamma=1 / 4$. This phenomenon had been reported, to a lesser extent, for unidirectional random fiber packings.
\end{abstract}

\section{INTRODUCTION}

Gas transport inside porous media is involved in many engineering applications, like particle filtering, catalytic and noncatalytic heterogeneous reactions, and various deposition processes. Especially, Chemical Vapor Infiltration (CVI) is a process designed to fabricate ceramic-matrix composite materials densifying a heated fibrous preform by the cracking of a gas and deposition reactions at the walls of the porous structure [1]. This technique has motivated the present work, since its modeling requires a sound knowledge of gas transport by diffusion inside possibly complex porous structures. In this paper attention is focused on Monte-Carlo techniques for simple reasons:

- In CVI conditions, gases are so rarefied that the free-molecule transport is not negligible compared to ordinary diffusion or forced convection. This is measured with the Knudsen number, $K n=\langle\lambda\rangle /\langle d\rangle$, where $\langle\lambda\rangle$ is the mean-free path between two successive molecule-molecule collisions and $\langle d\rangle$ is the mean pore diameter (which is of the order of magnitude of the mean distance between two successive moleculewall collisions). In the Knudsen or free-molecule regime, it looks natural to follow individually the trajectory of one particle that goes essentially from one wall to another.

- The computer capabilities now available allow one to handle a sufficient number of molecules during a long enough time to extract correct average coefficients from their behavior, the advantage being that the elementary rules are very simple to program.

According to the mean-square-displacement Monte-Carlo method, the effective orientation-averaged diffusivity of a gas in a porous medium, whatever the Knudsen number, is obtained by computing the 
mean-square displacement $\left\langle\xi^{2}\right\rangle$ of a large number of molecules travelling at mean thermal speed $c$, in the limit of large times [2-5]:

$$
D=\lim _{t \rightarrow \infty} \frac{\left\langle\xi^{2}\right\rangle}{6 t}
$$

Following the projections of the displacements on the main directions $\xi_{x}, \xi_{y}$ and $\xi_{z}$, one obtains the diagonal terms of the dispersion tensor:

$$
D_{i i}=\lim _{t \rightarrow \infty} \frac{\left\langle\xi_{i}{ }^{2}\right\rangle}{2 t} \quad(i=x, y \text { or } z)
$$

The results are usually presented under the form of a tortuosity factor, defined as follows. If we consider the ideal case of a packing of straight, parallel, non-overlapping cylindrical pores of constant diameter $\langle d\rangle$, an analytical solution for the orientation-averaged Knudsen diffusivity exists :

$$
D_{K n}=1 / 3 c .<d>
$$

On the other hand, if the Knudsen number is very small (ordinary diffusion regime), the selfdiffusivity of the gaseous species is given by:

$$
D_{b}=1 / 3 c .<\lambda>
$$

If the porosity $\varepsilon$ (defined as the ratio of void space to total space) is known, the averaged result over the packing of cylinders is simply $\varepsilon D_{K n}$ for Knudsen diffusion and $\varepsilon D_{b}$ for self-diffusion. Bosanquet [6] suggested that in the intermediate regime, the collision frequency is simply additive, i. e. :

$$
1 / l=1 /\langle\lambda\rangle+1 /<d\rangle
$$

where $l$ is the mean-free-path in the intermediate regime, and thus the effective diffusivity in the transition regime is also derived from a reciprocal additivity law :

$$
D_{m}^{-1}=D_{b}^{-1}+D_{K n^{-1}}
$$

The orientation averaged effective coefficient arising from this approximation is naturally $\varepsilon D_{m}$. If we consider that the mean pore diameter of a medium is obtained through the relation [7] :

$$
<d>=4 \varepsilon / S
$$

where $S$ stands for the specific surface, it is possible to compare the Monte-Carlo results in a given geometry with an equivalent ideal case (where all pores are straight). So, the tortuosity $\eta$ is defined as the ratio between the latter and the effective diffusion coefficient $D_{e f f}$, as it appears from the simulations :

$$
\eta=\left(\varepsilon D_{m}\right) / D_{\text {eff }}
$$

In this way, the $\eta$ factor contains almost all non-ideality effects due to the complex geometry. One defines similarly $\eta_{x}$ using the reference diffusivities along $x$, and so on.

\section{COMPUTATIONAL PROCEDURE}

\section{1.Trajectory handling.}

The procedure chosen in this work is directly inspired from former papers [3,5]. However, two problems have been adressed in more detail in the present work:

- When there is a molecule-wall collision, then new random directions are generated with isotropic distribution until one of them is indeed emergent (this implies a prior determination of the outward normal to the wall). To avoid unphysical behavior of the particles in some ideal cases (e.g. straight square or cylindrical pore), the direction probability density is isotropic unless the chosen direction lies too close to the wall : in this case, it shrinks to zero when the scalar product of the emergent direction and the normal to the wall goes to zero. A threshold is chosen according to the local curvature error due to the discretization. This modification does not affect significantly the molecule-wall collision frequency statistics. 
- At each step, a test is made to determine whether the molecule reaches the boundaries of the 3D image sample, considered as a representative elementary volume (REV). Two sets of coordinates (and direction vectors) have to be followed separately : locai coordinates of the molecule inside the REV, and global coordinates, which are used for the computation of the total displacements. Various possibilities are used to determine the local coordinates after crossing a boundary : i) reflection (symmetry), ii) reentrance by the opposite face (translation), iii) random reintroduction in the void space (random translation), iv) reentrance at a random location of the void part of the opposite face. Condition $i v$ ) is particularly well suited to simulate random stackings of tissues.

The trajectory-handling procedure is schematically shown in figure 1. Of course, molecule-molecule and molecule-wall collision frequencies may be acquired separately.

\subsection{Representation of the porous media boundary.}

Along with the two questions quoted above, the problem of describing a porous medium through the use of a computer image has been adressed in more detail, in order to enhance the accuracy of the geometrical description of the porous medium boundary.

A computer-discretized 3D image is a 3-dimensional array of boolean values, e.g. 1 for black (solid), and 0 for white (void space). It is a discrete representation of the medium, i. e. one only knows actually if it is solid or not at the nodes of a network. The most traditional interpolation choice for any position outside these nodes is to consider that this position has the same color as the closest node. In the case of a simple cubic network, this is the "voxel" representation, where the space is discretized in small cubes either black or white [8-9]. The advantages of this method are evident in terms of computational costs. However, the arrors in approximating the normal to walls of an arbitrary complex shape are important. To overcome partially this point, a slightly better interpolation scheme has been used, based on a local determination of the walls inside a cube of size unity and whose summits are nodes of the discretized image .The particle is considered as going from cube to cube, any cube being defined by 8 nodes of the discretized representation of the image. If the nodes are not all black or all white, the black ones are split into subsets of nodes linked together by edges. When a subset contains three black points or more, then investigation is made of the closest triangle having effective intersection with the trajectory : it is considered as a wall of the porosity. Fig. 1 gives a 2D example of boundary determinations following this algorithm. The effect of this algorithm in $3 \mathrm{D}$ is to approximate the interface by a set of connected triangles with Miller indices $h k l$, where $h, k$, and $l$ may independently take the values $-1,0$ and 1 (for a total of 13 distinct plane orientations only, since $e . g .001$ and $00 \bar{l}$ are undistinguishable), while the usual voxel interpretation restricts the choice to only three distinct orientations 100,010 and 001 .

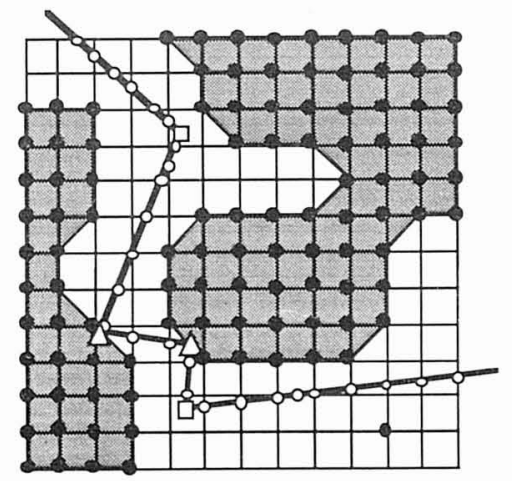

Legend :

\begin{tabular}{|cl|}
\hline - & Black pixels of the discretized image \\
$\circ$ & Step towards next elementary cube \\
$\square$ & Step towards next molecule-molecule collision \\
$\triangle$ & Step towards next wall-molecule collision \\
\hline
\end{tabular}

Fig. 1. : Scheme of the discretization with the "triangles" method and of the trajectory handling algorithm for molecules in transition diffusion regime. 
A comparison with the voxel interpretation method has been carried out on the simple example of a straight cylindrical pore with diameter $d_{h}=30$ voxel edges, where one molecules diffuses in the pure Knudsen regime (i. e., it undergoes only molecule-wall diffuse collisions) for at least 34,000 collisions. Table 1 shows the results for the a priori estimation of the hydraulic diameter, and the computed mean intercept lengths $\langle d\rangle$, using either the analytic description of the cylinder (no discretization), or the two interpretation schemes. One sees readily that the voxel method yields a much smaller $d_{h}$ and $\langle d\rangle$ than expected : this is mainly due to an important overestimation of the pore cross section perimeter. On the other hand, the triangle method gives excellent results in comparison with no discretization. Fig. 2 shows the free-path distributions for about 3,400 collisions. Both methods lead to an important difference in the distribution in the region of small $d$, which is due to the masking effect of the steps : one molecule hitting a wall close to a step experiences more quickly a second collision than when the surface is smooth. Of course, the steepness of the steps in the voxel interpretation is more important, thus leading to a more consequent error than with our method. Table 1 also shows the error between the computed distributions for the two methods and the non-discretized one, defined as :

$$
\mathrm{E}=\int_{0}^{\infty}\left|\mathrm{P}_{\text {comp }}(d)-\mathrm{P}_{\mathrm{nd}}(d)\right| \mathrm{d} d
$$

Again, the triangle method yields a lesser error.

Table 1 : Comparison of the results obtained with the voxel and triangle methods for pure Knudsen diffusion inside a zylindrical pore of diameter $d_{h}=30$.

\begin{tabular}{|c|c|c|c|c|c|}
\hline & $\begin{array}{c}\text { Hydraulic diameter } \\
d_{h}=4 \varepsilon / S \text { (vox.) }\end{array}$ & $\begin{array}{c}\text { Mean intercept } \\
\text { length }<d> \\
(\text { vox. })\end{array}$ & $\begin{array}{c}\text { Rel. error } \\
\text { w/ prediction } \\
<d>/ d_{h}(\%)\end{array}$ & $\begin{array}{c}\text { Rel. error } \\
\text { w/ no } \\
\text { discretization } \\
\text { and } d_{h}=30 \\
(\%)\end{array}$ & $\begin{array}{c}\text { Distribution } \\
\text { error } \\
(\text { eq. 9) }\end{array}$ \\
\hline No discretization & 30 & $29.49 \pm 0.5$ & -1.7 & - & - \\
\hline Voxel method & 23.096 & $23.97 \pm 0.5$ & +3.8 & 20,1 & -0.518 \\
\hline Triangle method & 29.501 & $29.502 \pm 0.5$ & +0.03 & 1,7 & -0.372 \\
\hline
\end{tabular}

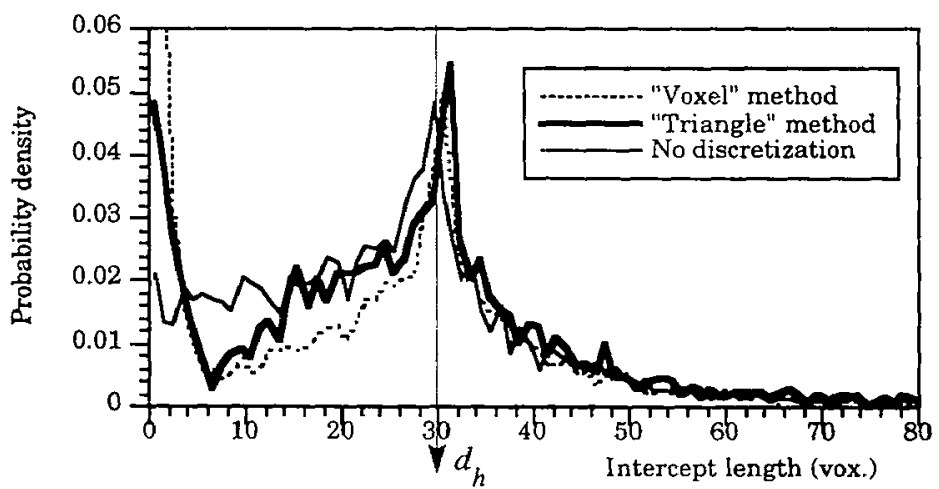

Fig. 2: Comparison of the free-path distribution function in pure Knudsen regime inside a straight cylindrical pore.

Although this test shows an excellent enhancement in the geometrical description through the triangle method, several disadvantages have to be quoted :

- The method is not symmetric towards an inversion of color, i. e., inverting black and white pixels does not always lead to the same approximation of the interface.

- The computational time is $50 \%$ higher than when working with the voxel interpretation. However, it remains acceptable on usual workstations. 


\section{EFFECTIVE DIFFUSIVITIES IN TISSUES.}

\subsection{A Modelization of a stacking of tissues.}

Investigation has been made of the diffusivities in idealized tissues, $i . e$. computer-generated 3D images made out of a simple analytical description as schematized in fig. 3, where the bundles are generated from a sinusoidal line with an elliptic cross section. It appears readily that this image depends only on five parameters :

- The horizontal $(L \times L)$ and vertical $(H)$ dimensions of the image, which are related to the horizontal spacing of the bundles, and the vertical spacing of the layers ;

- The amplitude $A$ of the bundle sinusoid;

- The major and minor radii $r_{l}$ and $r_{2}$ of the elliptic section of a bundle. Usually, $r_{I}$ is the largest radius, since in a true stacking of tissues, there is a vertical compression of the bundles due to the sewing and stacking processes.

For sake of simplicity, these 5 degrees of freedom have been reduced by application of a condition of mechanical stability of the tissue, i.e., the bundles must have a contact surface between each other in the $\mathrm{z}$ direction. This also ensures that the represented medium does not contain infinite slabs of void space, where the particules could "percolate towards the infinite" and lead to a divergence in the Knudsen diffusion coefficients. Accordingly, one has the following constraining relations :

$$
A=2 r_{2} \geq H / 2
$$

If the inequality gets strict, there is a nonzero area of contact between the bundles of two layers. It has been chosen to give a fixed 0.1 pixel excess in $A$ relatively to $H / 2$. Thus, only three degrees of freedom remain to describe the tissue, e.g., $H, L$, and $r_{l}$, which can be further reduced to two by scaling on $L$. In the following, only variations of $H / L$ and $r_{I} / L$ will be inspected.
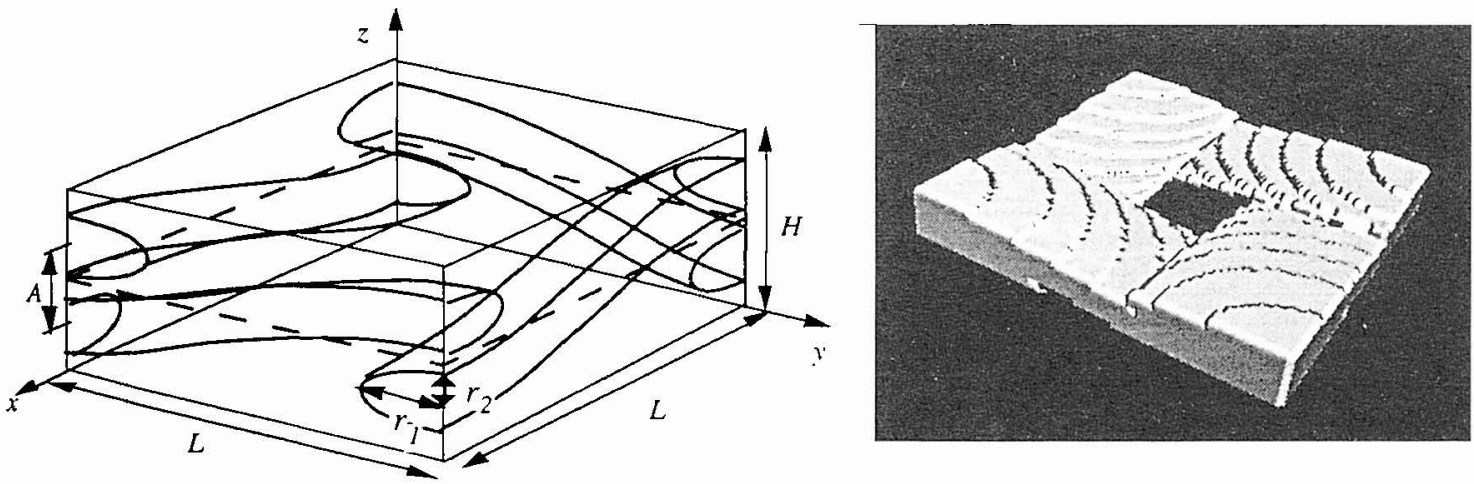

Fig. 3 : Idealized representative elementary volume (REV) for a tissue layer: a) Geometric parameters. b) 3D view of a discretized REV.

Applying a symmetry boundary condition in $x$ and $y$ to the REV leads to a $2 \mathrm{D}$ infinite layer of tissue, and the stacking of the layers may be monitored by applying different boundary conditions in $z$. It has been chosen to study the cases given by conditions $i$ ) and $i v$ ) (see section 2.1). If condition i) (symmetry) is used, then all the layers lie exactly one over each other, thus creating large, periodically constricted pores parallel to the $z$ direction. On the other hand, if condition $i v$ ) is used, giving a more realistic representation of a true stacking of tissue like a $2 D$ composite material preform, then these pores disappear or display a large tortuosity. 


\subsection{Computational results and discussion.}

All the computations presented here have been carried out with 1000 molecules travelling approximately 500 times the hydraulic diameter, though convergence is usually attained for lower times. Even after having converged, the results still display a dispersion of 8 to $10 \%$ and more for high tortuosities $(i$. e. lou diffusivities), and low Knudsen numbers. However, the accuracy of the results is sufficient to detect the main trends of evolution.

\subsubsection{Evolution of the diffusivities with the stacking mode of the tissues.}

Computations have been carried out on a single image sample $\left(L=80, H=12, A=6.1, r_{I}=32, r_{2}=3.05\right.$ voxel edges) shown in fig. $3 \mathrm{~b}$ ). Three kinds of tissue stackings have been modelled : 1) a symmetrical stacking, through application of boundary condition i),2) a stacking in opposition, obtained by creating an image with a double unit cell, and 3) a random stacking, through application of b. c. type iv). The results are shown in Table 2. The most important difference between these media lies in the stacking direction $z$ : the tortuosity in the pure Knudsen regime $\eta_{\perp} K$ is much more important, as one could expect, for tissues stacked in opposition than when the holes match each other. The random stacking appears to be an intermediate situation, its tortuosity $\eta_{\perp}{ }^{K}$ being roughly the arithmetic mean between the two other cases. This allows one to suggest that one of the most important characteristics of a stacking of tissues with respect to Knudsen diffusion is the amount of matching holes between different layers.

Table 2 : Knudsen regime tortuosities in different stackings of the same tissue layer.

\begin{tabular}{|l|l|l|l|}
\hline Stacking type & $\eta_{\prime^{K}}$ & $\eta_{\perp}{ }^{K}$ & $\left\langle\eta^{K}>\right.$ \\
\hline Symmetry & 0.88 & 6.37 & 1.23 \\
\hline Opposition & 0.67 & 22.41 & 0.98 \\
\hline Random & 0.63 & 12.85 & 0.93 \\
\hline
\end{tabular}

\subsubsection{Evolution of the diffusivities with the geometrical parameters of the tissue.}

The evolution of the geometric parameters $r_{l}$ and $H$ (using constant $L$ ) has been followed, in both limits of pure Knudsen and pure binary regimes, and using either symmetric or random stacking. Results are shown in figs. 4 and 5. Fig. 4 shows that $r_{l}$ affects mostly the perpendicular tortuosity, the section of the holes decreasing when $r_{l}$ increases. From fig. 3a), it appears that the percolation threshold lies at $r_{l}=L / 2$ (at least for low enough values of $H / L$ ). Accordingly, it looks rather normal that $\eta_{\perp}$ increases with $r_{l}$, and becomes less different for the two stacking modes as $r_{l}$ increases. The tortuosity factors in the pure binary limit show a lesser dependence on $r_{l}$, in agreement with the fact that the molecules in the binary diffusion regime should feel much less the effect of the geometric details of the interface than in the Knudsen limit.

On the other hand, fig. 5 shows a different evolution for $\eta_{\perp}{ }^{K}$ with $H$ depending on the stacking mode : indeed, if the holes match, the effect of decreasing $H$ is to make the medium look more and more like a dispersion of straight pores with diameter equal to the hole size. If they do not match, the unmatching effect is augmented by the closeness of the layers in the $z$ direction : an imaginary path passing by two holes in opposition (representing a tortuous pore) is more curved when the holes are close. Again, as expected, this feature is not present in the binary diffusion regime. 

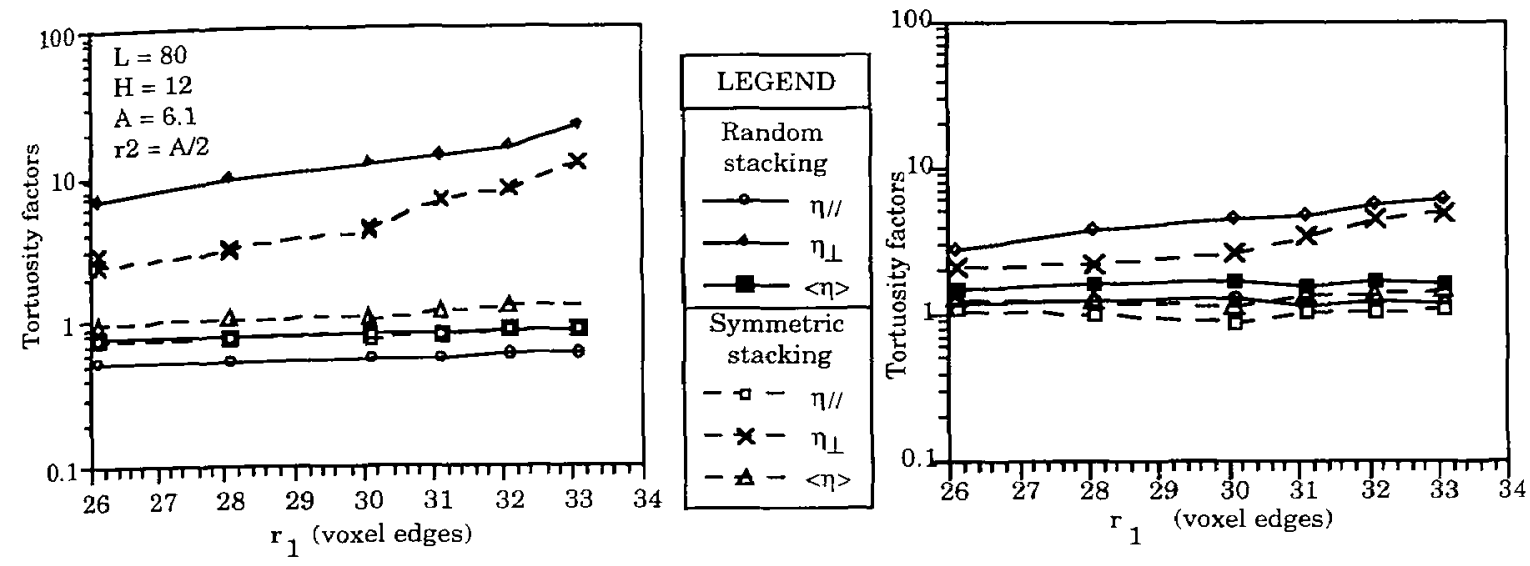

Fig. 4 : Evolution with the bundle radius $r_{l}$ of a) the Knudsen tortuosity, b) the binary regime tortuosity.
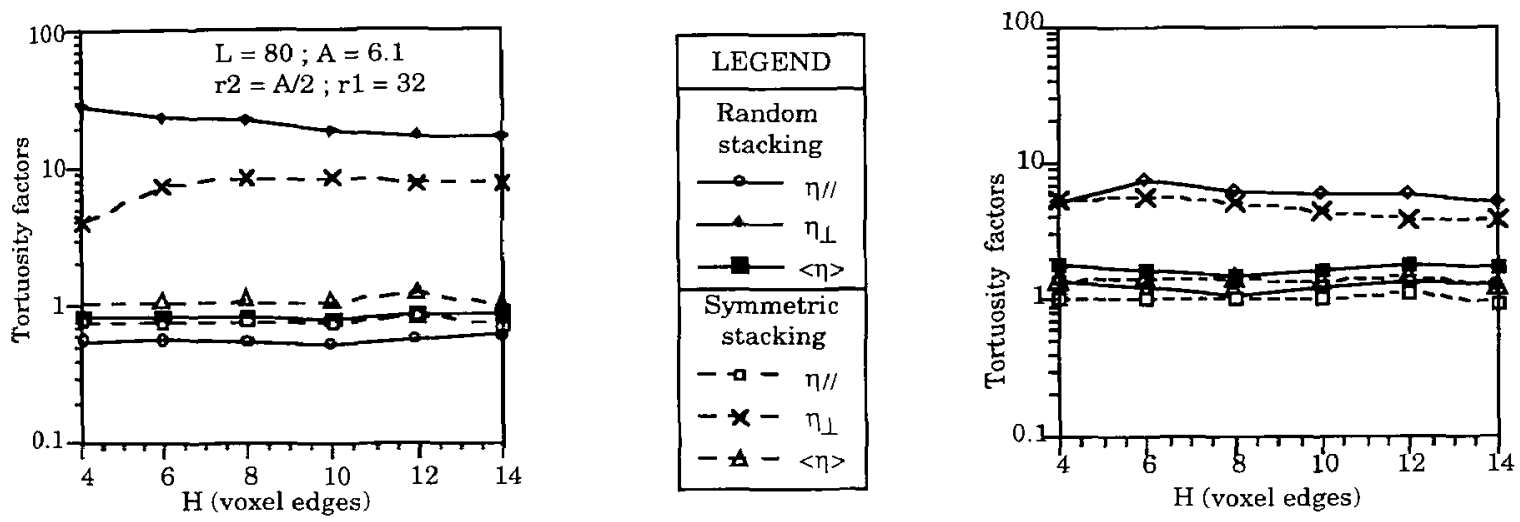

Fig. 5 : Evolution with the vertical size $H$ of a) the Knudsen tortuosities, b) the binary regime tortuosities.

\subsubsection{Intermediate regime diffusion}

Computations have been carried out on the first image sample (see subsection 3.2.1), with symmetry boundary conditions. The results are plotted as tortuosity factors in fig. 6 . It appears from them that a neat tortuosity variation occurs for $0.1<K n<100$. This is not in agreement with results obtained for many porous media $[3,4,5]$, where the transition regime is reported to occur for $0.01<K n<100$. The tortuosity $\eta$ and the components parallel and perpendicular to the tissue planes, $\eta_{/ /}$and $\eta_{\perp}$, vary more or less monotonically between two limits, $\eta^{K}$ for the Knudsen regime and $\eta^{b}$ for the binary regime. The intermediate values may be fitted to the following law [11]:

$$
\eta(K n)=\frac{\eta^{b}+\eta^{K} \gamma K n}{1+\gamma K n}
$$

A $92 \%$ correlation for $\eta$ is obtained by taking $\gamma \approx 0.25$. In the case of random unidirectional fiber structures [11], $\gamma$ has been found to be 0.6 , and it is unity in numerous random fiber and cylinder structures - so $\gamma=1$ 
appears to be the usual case. When $\gamma \neq 1$, the medium behaves as if the most characteristic length of its geometry were $d_{h} / \gamma$ instead of $d_{h}$. No clear explanation of this phenomenon has been reported.

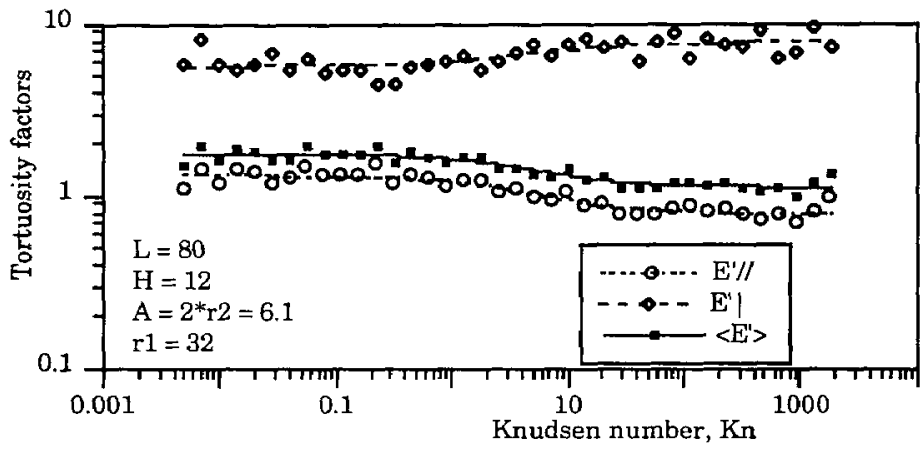

Fig. 6: Intermediate diffusion regime in a symmetric tissue stacking. The curves are from eq. (11).

\section{Conclusion}

A Monte-Carlo mean-square-displacement method has been designed to study the binary, Knudsen and transition regime diffusivities inside complex porous media, as represented by discrete 3D computer binary images through a special interpretation. This procedure gives better approximations to curved interfaces than the usual "voxel" interpretation. The program has then been used to study the diffusivity inside stackings of tissues. Two features appear from the study. First, the diffusivity perpendicular to the layers varies greatly according to the size and relative position of the holes present in the tissue layers. Second, the intermediate regime diffusivities parallel and perpendicular to the tissue layers do not follow the simplest Bosanquet law, owing to anisotropic geometry effects.

Further developments include the study of 3D images acquired by X-ray microtomodensitometry of porous media, and the extension of the transport simulation to the coupling of diffusion and forced convection.

\section{Acknowledgements}

This work has been supported by SEP as part of a more general approach to the modeling of the CVI of complex fibrous preforms.

The author is indebted to his referee's numerous comments.

\section{References}

[1] Naslain, R. and Langlais, F., Mat. Sci. Res. 20 (1986) 145.

[2] Chandrasekhar, S., Rev. Mod. Phys. 15 (1943) 1.

[3] Burganos, V. N. and Sotirchos, S. V., Chem. Eng. Sci. 44 (1989) 2451.

[4] Burganos, V. N. and Sotirchos, S. V., Chem. Eng. Sci. 44 (1989) 2629.

[5] Tassopoulos, M. and Rosner, D. E., Chem. Eng. Sci. 47 (1992) 421.

[6] Pollard, W. G. and Present, R. D., Phys. Rev. 73 (1948) 762.

[7] Satterfield, C. N., Mass transfer in heterogeneous catalysis (MTT Press, Cambridge, 1970).

[8] Sallès, J., Thovert, J. F. and Adler, P. M., Chem. Eng. Sci. 48 (1993) 2839.

[9] Quintard, M., and Whitaker, S., Chem. Eng. Sci. 48 (1993) 2537.

[10] Tomadakis, M. M. and Sotirchos, S. V.,AlChE J. 39 (1993) 397. 